[Contributions from the Chemical Laboratory of Harvard College.]

\title{
I-BENZOYLPHENYL-3-METHYL-5-PYRAZOLONE. ${ }^{1}$ \\ BY HENRY A. TORREY AND H. R. RAFSEY. \\ Received A ugust 27, 1910.
}

Pyrazolones containing a radical other than phenyl in the I-position are far from common, and in the present paper we add another member to this small class of compounds. The I-benzoylphenyl-3-methyl-5pyrazolone was made (following Michael's general method for making pyrazolones) from the hydrochloride of parahydrazinobenzophenone and acetoacetic ester with a few drops of hydrochloric acid. It melts at $170-171^{\circ}$. Uniortunately, the short time at our disposal prevented us from studying, beside the free base, more than the hydrochloride and the dimethyl compound, of which latter we did not get an analysis. We add an improvement to Döbner's method of making paraminobenzophenone, and several details which we found necessary to insure success in Ruhemann and Blackman's method of making the parahydrazinobenzophenone.

\section{Experimental Part.}

The paraminobenzophenone necessary for this work was made according to the method of Döbner ${ }^{2}$ by the action of benzoyl chloride on phthalanil in presence of zinc chloride (the purified product being subsequently saponified into the paraminobenzophenone), but we found that the hard, pitch-like substance formed could be worked up with better results by extraction with alcohol, without boneblack, than by the treatment with glacial acetic acid and boneblack recommended by him. For this purpose several hundred grams of the crude substance were boiled with a reflux condenser for some time with 700-800 cc. of alcohol, the solution filtered boiling through a steam funnel, and the filtrate cooled in ice water, when crystals were deposited. By using two portions of alcohol the process could be run continuously, and, although it took more time than that with acetic acid, it gave much less trouble, and the product, while not quite so pure, proved to be sufficiently so for our purpose The paraminobenzophenone prepared from it with potassium hydroxide, after one recrystallization, melted at $\mathrm{I} 24^{\circ}$, as it should; but the principal advantage of this method was that the yield was $70-180$ grams as compared with 7o-80 grams by the old method.

Parahydrazinobenzophenone was prepared by the method of Ruhemann and Blackmann, ${ }^{3}$ consisting in the reduction of the diazo com-

${ }^{1}$ This research was suggested by the late Professor $\mathrm{H}$. A. Torrey, and somewhat more than half of the experimental work was done under his direction, but the remainder of the experimental work and the writing of the paper were deprived of the benefit of his supervision by his too early death.

C. L. JACKSON.

2 Ann., 210, 267.

'J. Chem. Soc., 55, 6r3. 
pound with stannous chloride and hydrochloric acid. This process, as was to be expected, gave us much trouble, but we finally succeeded in obtaining the hydrazine by observing the following precautions: The paraminobenzophenone was ground in a mortar with strong hydrochloric acid, so as to obtain a powder fine enough to remain easily in suspension in the hydrochloric acid used for the diazotization. The mixture was cooled carefully with ice and the concentrated solution of sodium nitrite added in five portions at intervals of an hour with frequent shaking. The completion of the reaction was determined by the disappearance of yellow sediment, but a white one always remained.

The solution was allowed to stand overnight in ice, filtered, and added slowly, with constant stirring, to the solution of stannous chloride in strong hydrochloric acid (which was also well cooled with ice) at which the yellow precipitate mentioned by Ruhemann and Blackman was formed. This, after standing in the cold solution for half an hour, was filtered and dried on a porous plate. If it was dried on a steam radiator, it turned red, but this red product seemed to give the hydrazine hydrochloride as well as the orange-yellow one which was dried at ordinary temperatures.

The extraction of the hydrochloride from this product gave much trouble. The first extraction with a large amount of warm water could be filtered off without difficulty, but the second converted the residual tin salt into the colloidal state, when it was impossible to remove it by filtering, or decantation. After many experiments this difficulty was overcome as follows: The crude product was warmed with twice its volume of water at $50-60^{\circ}$ with shaking for several minutes. As stated by Ruhemann and Blackman, heating to boiling causes some decomposition. It was then filtered rapidly, and by quick work a second similar extraction could be carried through before the precipitate had grown too fine to filter, but, when water was added for the third extraction, a colloidal solution was formed. By the addition of hydrochloric acid to this colloidal solution a precipitate similar to the original was formed which, after being filtered, and pressed on a porous plate, could be extracted three times before it became colloidal, and fortunately this was enough to extract all the hydrochloride of the hydrazine. This was precipitated from the combined water extracts by the addition of hydrochloric acid. The free hydrazine was then obtained by the addition of a concentrated solution of sodium acetate to a warm aqueous solution of the hydrazine hydrochloride. We have given an account of these additional details of the process in the hope of saving others the large expense of time and labor, which we found necessary in order to get a good result.

I-Benzoylphenyl-3-methyl-5-pyrazolone,

$\mathrm{C}_{6} \mathrm{H}_{5} \mathrm{COC}_{6} \mathrm{H}_{4}-\mathrm{N}-\mathrm{N}=\mathrm{C}\left(\mathrm{CH}_{3}\right) \mathrm{CH}_{2}$. - As the method of $\mathrm{Knorr}^{1}$ for the production of pyrazolones seemed to give a very impure product in this 
case, we tried to prepare the substance by the general method of Michael, ${ }^{1}$ which also had the advantage of using the hydrochloride, and thus avoiding the somewhat wasteful preparation of the free hydrazine. The hydrochloride of the parahydrazinobenzophenone mixed with acetoacetic ester in molecular proportions (a slight excess of the ester does no harm) and a few drops of hydrochloric acid were heated on the steam bath for half an hour. The mixture melted, gave off vapors at first, and was converted into a yellow, pasty mass, which solidified on cooling. It was purified by crystallization from alcohol until it showed the constant melting point I $70-171^{\circ}$.

The analyses gave ro. $3 \mathrm{I}$ and Io.or per cent. $\mathrm{N}$; calculated for $\mathrm{C}_{17} \mathrm{H}_{14} \mathrm{O}_{2} \mathrm{~N}_{2}$, gives 10.07 per cent. N.

Properties of I-Benzoylphenyl-3-methyl-5-pyrazolone.-Brownish yellow crystals from alcohol, which melt at $170-171^{\circ}$. Freely soluble in alcohol; rather soluble in benzene; very slightly soluble in ether; almost insoluble in cold, slightly soluble in hot water. Dissolves on warming in strong hydrochloric acid or in an aqueous solution of sodium hydroxide, if not too concentrated. Silver nitrate gives a white flocculent precipitate with an alcoholic solution. The pyrazolone does not reduce Fehling's solution.

Hydrochloride.-The I-benzoylphenyl-3-methyl-5-pyrazolone was dissolved in benzene and dry hydrochloric acid gas was passed into the solution. The light brown precipitate formed was dried in vacuo.

The analysis gave II . 55 per cent. $\mathrm{Cl}$; calculated for $\mathrm{C}_{17} \mathrm{H}_{34} \mathrm{O}_{2} \mathrm{~N}_{2} \mathrm{HCl}$, gives II 27 per cent. Cl.

We obtained the salt as a pale brown powder, but the color may have been due to a trace of impurity. At $180^{\circ}$ it turns dark, shrivels up at $193^{\circ}$, and melts with decomposition at $196^{\circ}$.

In an attempt to make the 2-3-dimethylpyrazolone corresponding to antipyrine, a method similar to Knorr's ${ }^{2}$ was used. Three grams of I-benzoylphenyl-3-methyl-5-pyrazolone were mixed with 3 grams of methyl iodide and 3 grams of methyl alcohol, and the mixture heated to $100^{\circ}$ in a sealed tube for 16 hours. The product, a thick dark brown liquid, was warmed on the steam bath and treated with sulphurous anhydride, which hardened the mass, but produced no perceptible decolorization. After cooling and pulverization it was shaken with a very strong sodium hydroxide solution and then dissolved in benzene; after standing for several days the evaporation of the red-brown solution left an amorphous mass with a few embedded crystals; these were removed and used to inoculate the benzene solution of the amorphous portion, and in this way crystals were obtained which, after a second crystallization (with inoctlation), were nearly white. Not enough of this substance, could be obtained for analysis, because the end of the year prevented a second preparation, but

1 Am. Chem. J., 1892, 517. 
there seems no question that it is the I-benzoylphenyl-2,3-dimethyl-5-pyrazolone, from the method of its formation and the fact that ferric chloride gives a dark red color with its aqueous solution. It is very soluble in alcohol, or benzene; somewhat soluble in cold, soluble in hot water; soluble in strong hydrochloric acid. The melting point obtained by us ( $125^{\circ}$ ) was probably too low, as the amount at our disposal was too small to make certain of the purity of our specimen.

[Contributions from the Chemical Laboratory of Harvard College.]

NOTE ON SOME PROPERTIES OF PIPERONYLOIN.

BY HENRY A. TORREY AND J. B. SUMNER. 1

Received August 27 , I9I0.

The object of this work was to compare the behavior of piperonyloin and benzoin under similar conditions. The number of results obtained is small, partly because we had but little time at our disposal, but still more because the piperonyloin, $\mathrm{CH}_{2} \mathrm{O}_{2} \mathrm{C}_{8} \mathrm{H}_{3} \mathrm{COCHOHC} \mathrm{C}_{6} \mathrm{H}_{3} \mathrm{O}_{2} \mathrm{CH}_{i}$, proved to be much less reactive than benzoin; thus no substance corresponding to desoxybenzoin could be obtained under the usual conditions for forming that substance, or a variety of modifications of them. Most of the piperonyloin remained unaltered, the product of the reaction being little more than a trace of what seemed to be a pinacone. No oxime could be made: in fact there was no reaction, as far as we could find, under conditions which give the oxime of benzoin with ease; and no more than a trace of an acetyl compound was formed by long continued and varied action of acetyl chloride on the piperonyloin. These differences between benzoin and piperonyloin form the most important result of this research, but until more observations have been made, it is not worth while to speculate on their cause.

The only substances we have found which react easily with piperonyloin are urea and ammonium thiocyanate. The ureide decomposes at $262^{\circ}$ the thioureide near $260^{\circ}$, but the formula of the latter has not been established by us with certainty.

We also describe a modification of Perkin's method of preparing piperonyloin, which makes it possible to use larger quantities of substance.

\section{Experimental Part.}

The piperonyloin used in this work was prepared by the method of Perkin, ${ }^{2}$ but we found that we obtained a better result; when instead of heating the mixture of 5 grams piperonal, 20 grams of 50 per cent. alcohol, and 2 grams of potassium cyanide for 6-8 hours, as recommended by

1 This research was suggested by the late Professor H. A. Torrey, and somewhat more than half of the experimental work was done under his direction, but the remainder of the experimental work and the writing of the paper were deprived of the benefit of his supervision by his too early death.

J. Chem. Soe., $59, \mathrm{j}^{164}$.

C. L. JACRSON. 